\title{
Exploratory Research on the Relationship between Human Gut Microbiota and Portal Hypertension
}

\author{
Keiji Yokoyama, Naoaki Tsuchiya, Ryo Yamauchi, Takashi Miyayama, Yotaro Uchida, \\ Kumiko Shibata, Hiromi Fukuda, Kaoru Umeda, Kazuhide Takata, Takashi Tanaka, \\ Shinjiro Inomata, Daisuke Morihara, Yasuaki Takeyama, Satoshi Shakado, \\ Shotaro Sakisaka and Fumihito Hirai
}

\begin{abstract}
:
Objective The relationship between gut microbiota and portal hypertension remains unclear. We investigated the characteristics of the gut microbiota in portal hypertension patients with esophago-gastric varices and liver cirrhosis.

Methods Thirty-six patients (12 patients with portal hypertension, 12 healthy controls, and 12 non-cirrhosis patients) were enrolled in this university hospital study. Intestinal bacteria and statistical analyses were performed up to the genus level using the terminal restriction fragment length polymorphism method targeting $16 \mathrm{~S}$ ribosomal RNA genes, with diversified regions characterizing each bacterium.

Results Levels of Lactobacillales were significantly higher $(\mathrm{p}=0.045)$ and those of Clostridium cluster IV significantly lower $(\mathrm{p}=0.014)$ in patients with portal hypertension than in other patients. This Clostridium cluster contains many butanoic acid-producing strains, including Ruminococcace and Faecalibacterium prausnitzii. Clostridium cluster IX levels were also significantly lower $(\mathrm{p}=0.045)$ in portal hypertension patients than in other patients. There are many strains of Clostridium that produce propionic acid, and the effects on the host and the function of these bacterial species in the human intestine remain unknown. Regarding the Bifidobacterium genus, which is supposed to decrease as a result of cirrhosis, no significant decrease was observed in this study.

Conclusion In the present study, we provided information on the characteristics of the gut microbiota of portal hypertension patients with esophago-gastric varices due to liver cirrhosis. In the future, we aim to develop probiotic treatments following further analyses that include the species level, such as the intestinal flora analysis method and next-generation sequencers.
\end{abstract}

Key words: gut microbiota, portal hypertension, esophageal and gastric varices, liver cirrhosis

(Intern Med 59: 2089-2094, 2020)

(DOI: 10.2169/internalmedicine.4628-20)

\section{Introduction}

The human gut is inhabited by up to 100 trillion cohabiting bacterial groups. The intestinal bacteria make up an extensive metabolic system in which bacteria interact, and this is thought to have a major impact on the host's state of health. It has been reported that imbalance of the gut micro- biota, which is called dysbiosis, contributes to various diseases including inflammatory bowel disease, irritable bowel syndrome, colon cancer, nonalcoholic steatohepatitis (NASH), liver cirrhosis, hepatocellular carcinoma, diabetes, depression, and multiple sclerosis (1-4).

A recent $16 \mathrm{~S}$ ribosomal RNA gene and meta-genome analysis clarified the entire gut microbiota that has previously only been found by conventional culture methods, and 
Table 1. The Background Characteristics of the Study Population.

\begin{tabular}{lccc}
\hline & PH-G $(\mathrm{n}=12)$ & HC-G $(\mathrm{n}=12)$ & CP-G $(\mathrm{n}=12)$ \\
\hline Age $($ median \pm SD) & $64.1 \pm 11.4$ & $40.0 \pm 8.27$ & $71.7 \pm 13.0$ \\
Male : Female & $7: 5$ & $10: 2$ & $5: 7$ \\
Body Weight (median \pm SD) & $61.7 \pm 8.25$ & $57.8 \pm 8.85$ & $57.5 \pm 7.87$ \\
Etiology & Liver cirrhosis & Volunteer & Colon polyp \\
& (Viral hepatitis/ Alcohol/ Others: 4/6/2) & (Medical staff) & (adenoma) \\
Child-Pugh Score (A/B/C) & $(4 / 7 / 1)$ & & \\
\hline
\end{tabular}

SD: standard deviation, PH-G: portal hypertension group, HC-G: hepatocellular carcinoma group, CP-G: colon polyp group

research on the gut microbiota and various types of diseases is underway (5). In liver cirrhosis, elevated levels of minute endotoxins in the portal vein induce the production of various inflammatory cytokines via increased toll-like receptor 4 signaling, thereby promoting liver fibrosis $(6,7)$. One study using a rat model reported that the existence of gut microbiota promotes lymph duct and vascular development in the intestinal mucosa, and that this might contribute to the development of portal hypertension ( $\mathrm{PH})(8)$.

In humans, the relationship between gut microbiota and $\mathrm{PH}$ remains unclear. In previous reports, Coprococcus, Ruminococcaceae, Lachnospiraceae (which contributes to gut immunity via butyric acid production) and Faeclibacterium prausnitzii (which plays an anti-inflammatory role) were all found to demonstrate significantly lower levels in patients with liver cirrhosis (9). While patients with $\mathrm{PH}$ are presumed to present with the same tendency, the particular characteristics of the flora require further investigation. Current research has focused on the usefulness of rifaximin (a rifamycin-based nonabsorbable oral antibiotic), the development of new probiotic treatments, and fecal microbiota transplantation.

In the present study, using a terminal restriction fragment length polymorphism (T-RFLP) method targeting 16S ribosomal RNA genes, with diversified regions characterizing each bacterium, we conducted an exploratory study to examine the characteristics of the gut microbiota in $\mathrm{PH}$ patients with esophago-gastric varices due to liver cirrhosis.

\section{Materials and Methods}

Among the patients admitted to our department from March to December 2016, 36 patients were selected. The exclusion criteria were patients under 20 years of age, patients with concurrent liver cancer, patients who had taken medication (antibiotics and/or bowel medicine) for intestinal disorders in the prior 3 months, patients with no recurrence of liver cancer but with a history of tumor invasion of the portal vein, and patients who were found to be unsuitable for participation in the present study by their attending physician. There were 12 patients in the in-patient group who received treatment for esophago-gastric varices with liver cirrhosis (PH group; PH-G), 12 patients in the healthy control group with normal hepatic deviation enzymes and with- out liver cirrhosis (healthy control group; HC-G), and 12 patients without liver cirrhosis who were hospitalized for the treatment of colon polyps (colon polyp group; CP-G). Stool samples were collected from all patients after they provided their informed consent, and intestinal bacterial and statistical analyses were conducted at the genus level using the TRFLP method.

All fecal samples were collected before the procedure for esophageal varices in the PH-G. Each individual's living environment and dietary status were difficult to examine in detail. Intestinal bacteria analyses were outsourced to TechnoSuruga Laboratory (Shizuoka, Japan) (10). The background characteristics of the study population are shown in Table 1.

This study was conducted in compliance with the Declaration of Helsinki and the Ethical Guidelines for Medical Research of the Ministry of Health, Labor and Welfare. In conducting this study, ethical considerations were carefully considered, and patient consent was obtained after a full explanation of the study method. Information obtained through this study was not disclosed in any way that could identify the individuals who participated, and this was strictly controlled. Patient data were tabulated using connectable anonymization. The present study protocol was approved by the Ethics Committee of our hospital.

Statistical analyses were performed using the JMP software program, version 13.0 (SAS Institute, Cary, USA). Comparisons between groups were conducted using a $t$-test. $P$ values less than 0.05 were considered statistically significant. This study was an exploratory study, and the number of samples was small, so this statistical analysis did not require multiple comparisons. Therefore, false discovery rate (FDR) adjustment was not performed in this study.

\section{Results}

The intestinal bacteria analyses for each of the three groups using the T-RFLP method are shown in Table 2. In the present study, we found no statistically significant difference in the gut microbiota of the HC-G and CP-G. Therefore, we combined the HC-G and CP-G into the non-PH group (Non-PH-G), and we conducted a comparative analysis of the PH-G and the Non-PH-G. Table 3 shows the intestinal bacterial analyses of each group using the T-RFLP method. A comparison of gut microbiota between the two 
Table 2. A Comparison of the Gut Microbiota between the HC and CP Groups (median \pm SD).

\begin{tabular}{lccc}
\hline & HC-G & CP-G & p value \\
\hline Bifidobacterium & $7.491 \pm 1.984$ & $3.309 \pm 1.811$ & 0.135 \\
Lactobacillales & $2.523 \pm 1.780$ & $6.413 \pm 1.625$ & 0.122 \\
Bacteroides & $45.52 \pm 4.364$ & $49.09 \pm 3.983$ & 0.553 \\
Prevotella & $10.150 \pm 4.776$ & $4.340 \pm 4.360$ & 0.380 \\
Clostridium cluster IV & $5.891 \pm 1.312$ & $6.768 \pm 1.198$ & 0.627 \\
Clostridium cluster XIVa & $12.31 \pm 1.559$ & $11.51 \pm 1.423$ & 0.709 \\
Clostridium cluster IX & $7.869 \pm 2.451$ & $5.668 \pm 2.238$ & 0.515 \\
Clostridium cluster XI & $0.206 \pm 0.299$ & $0.598 \pm 0.273$ & 0.344 \\
Clostridium cluster XVIII & $2.023 \pm 0.356$ & $1.101 \pm 0.325$ & 0.070 \\
others & $5.115 \pm 2.030$ & $9.952 \pm 1.853$ & 0.094 \\
\hline
\end{tabular}

SD: standard deviation, HC-G: hepatocellular carcinoma group, CP-G: colon polyp group

Table 3. A Comparison of the Gut Microbiota between the PH and Non-PH Groups (median \pm SD).

\begin{tabular}{lccc}
\hline & PH-G & Non-PH-G & p value \\
\hline Bifidobacterium & $9.784 \pm 1.913$ & $5.210 \pm 1.413$ & 0.063 \\
Lactobacillales & $13.72 \pm 2.079$ & $4.644 \pm 1.535$ & $0.0013^{*}$ \\
Bacteroides & $40.48 \pm 3.426$ & $47.47 \pm 2.530$ & 0.1104 \\
Prevotella & $3.494 \pm 3.839$ & $6.981 \pm 2.835$ & 0.470 \\
Clostridium cluster IV & $2.979 \pm 1.050$ & $6.370 \pm 0.775$ & $0.014^{*}$ \\
Clostridium cluster XIVa & $10.24 \pm 1.802$ & $11.87 \pm 1.331$ & 0.472 \\
Clostridium cluster IX & $1.845 \pm 1.860$ & $6.668 \pm 1.374$ & $0.045^{*}$ \\
Clostridium cluster XI & $0.796 \pm 0.391$ & $0.420 \pm 0.289$ & 0.446 \\
Clostridium cluster XVIII & $0.982 \pm 0.348$ & $1.520 \pm 0.257$ & 0.223 \\
others & $6.928 \pm 1.752$ & $7.753 \pm 1.294$ & 0.707 \\
\hline *statistically significant difference & & \\
SD: standard deviation, PH-G: portal hypertension group, Non-PH-G: non-portal \\
hypertension group
\end{tabular}

groups is shown.

All figures show the results of comparisons that confirmed the significant difference or tendency in gut microbiota between the PH-G and Non-PH-G. Compared to the Non-PH-G, the PH-G showed the following results:

A) Significantly higher levels of the Lactobacillales order $(\mathrm{p}<0.01)$ (Fig. 1)

B) Significantly lower levels of Clostridium cluster IV (p=0.014) (Fig. 2)

C) Significantly lower levels of Clostridium cluster IX $(\mathrm{p}=0.045)$ (Fig. 3)

D) A non-significant tendency towards higher levels of Bifidobacterium ( $\mathrm{p}=0.063$ ) (Fig. 4)

Furthermore, a comparison of the gut microbiota between the alcohol group (ALC, n=6) and non-alcohol group (NonALC, $n=6$ ) in the PH-G is shown in Table 4. This comparison showed that levels of Bifidobacterium were significantly higher in the ALC group than in the Non-ALC group ( $\mathrm{p}=$ 0.042).

\section{Discussion}

In the current study, we found no statistically significant difference between the HC-G and CP-G. However, a previous systematic review comparing the bacterial profiles of normal colon (HC) and colon adenoma reported that the colon adenoma group had greater microbial flora diversity than the HC group. Furthermore, a taxonomic analysis revealed that the amounts of eight phyla (Firmicutes, Proteobacteria, Bacteroidetes, Actinobacteria, Chloroflexi, Cyanobacteria, Candidate-division TM7, and Tenericutes) were significantly different between normal colon and colon adenoma (10). A greater number of samples might have resulted in significant differences being detected between the HC-G and CP-G in the present study, as described above. In our study, we considered the two combined groups to be a consistent group of non-PH with various background characteristics.

We confirmed several differences in gut microbiota between the PH and Non-PH-Gs in this study. First, the levels of Lactobacillales in the PH group were significantly higher 


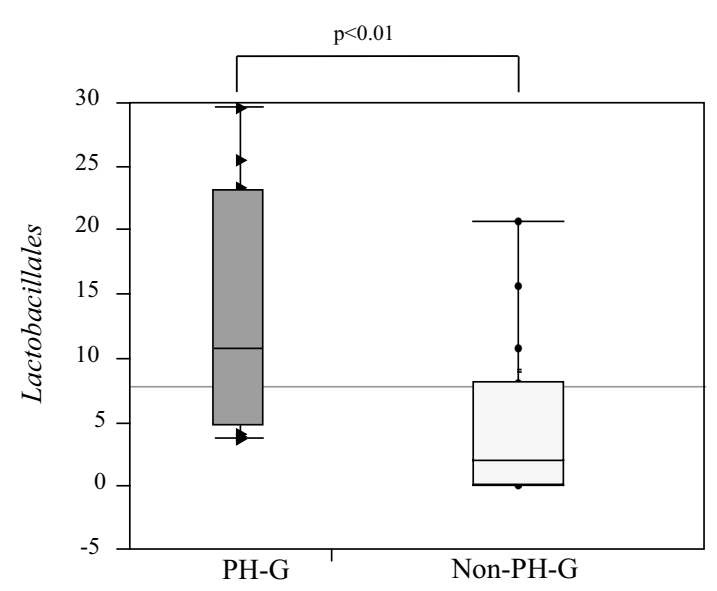

Figure 1. Significantly higher levels of the Lactobacillales order (p<0.01). PH-G: portal hypertension group, non-PH-G: non-portal hypertension group

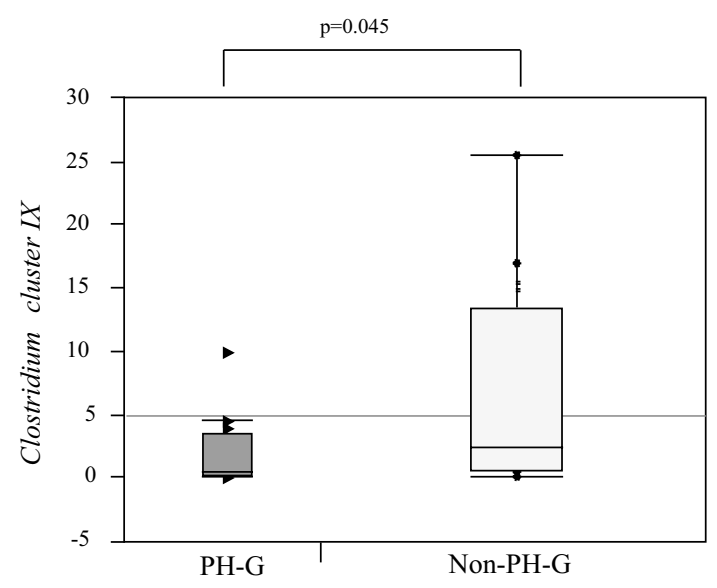

Figure 3. Significantly lower levels of Clostridium cluster IX ( $p=0.045)$. PH-G: portal hypertension group, non-PH-G: nonportal hypertension group

$(\mathrm{p}=0.045)$ than in the non-PH-G. The operational taxonomic unit of strains estimated from the base sequence and fragment length was elevated at 657, suggesting that the abundant Streptococcus genus (11), which is reported to be common in oral bacteria and elevated in liver cirrhosis, might be present. Among the 12 patients in the $\mathrm{PH}$ group, 11 were taking proton pump inhibitors (PPIs), so the relationship between the ongoing use of PPIs for the treatment of varices was also taken into consideration (12-15). It has been reported that the partial changes in the gut microbiota caused by PPI usage are similar to the changes caused by the progression of liver cirrhosis, and increased inflow of oral flora to the intestines might create an intestinal environment that is a risk factor for the progression of liver cirrhosis, spontaneous bacterial peritonitis, and hepatic encephalopathy (9,16-19). The levels of genera (Lactobacillales, Streptococcus, Selenomonas, Veillonella, Campylobacter, and Haemophilus) have been reported to be high in PPI users (20).

Second, Clostridium cluster IV levels were significantly lower $(\mathrm{p}=0.014)$ in the PH group than in the non-PH-G.

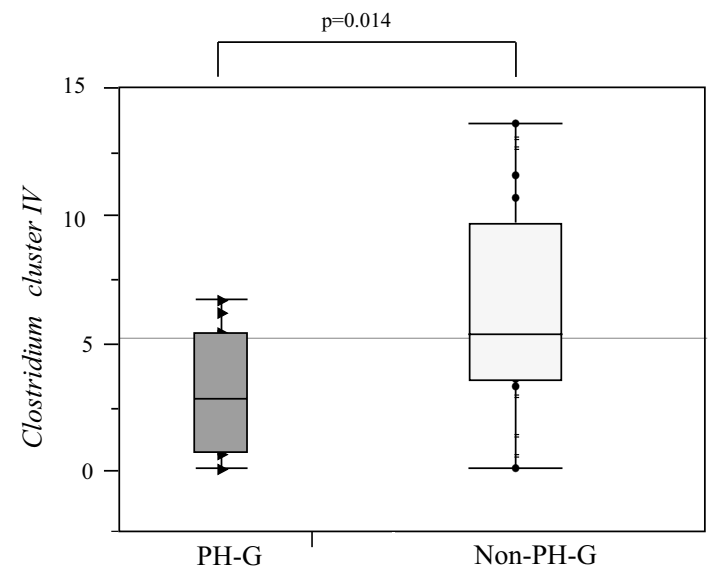

Figure 2. Significantly lower levels of Clostridium cluster IV ( $p=0.014)$. PH-G: portal hypertension group, non-PH-G: nonportal hypertension group

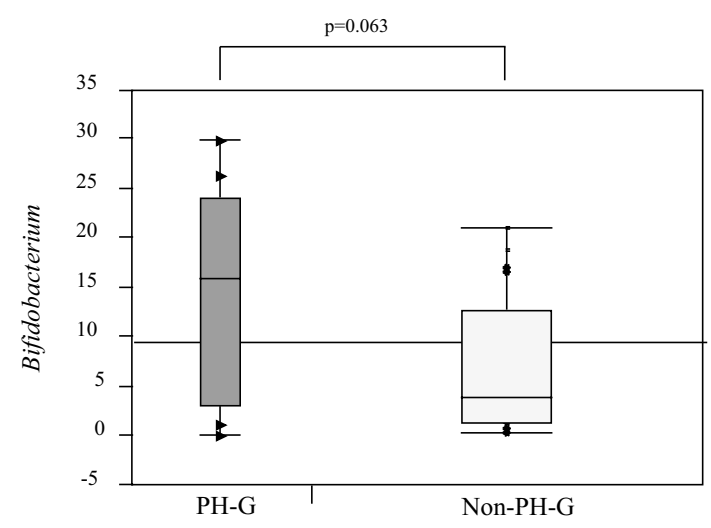

Figure 4. No significant differences in levels of Bifidobacterium ( $\mathrm{p}=0.063$ ). $\mathrm{PH}-\mathrm{G}$ : portal hypertension group, non-PH-G: non-portal hypertension group

This cluster has many strains that produce butyric acid, including Ruminococcaceae, which is reported to reduce liver cirrhosis, and F. prausnitzii, which plays an antiinflammatory role, as previously reported (21). Butyric acid bacteria and bacteria that produce short-chain fatty acids reportedly reduce the progression of liver cirrhosis and NASH $(22,23)$. In a report of mixed strains of Clostridium using abundant Clostridium cluster IV administered to mice, markedly increased levels of colonic regulatory $\mathrm{T}$ cells were confirmed (24).

Third, the Clostridium cluster IX levels were also significantly lower ( $\mathrm{p}=0.045)$ in the $\mathrm{PH}$ group than in the non-PHG. This cluster is said to have many strains that produce propionic acid; however, for most strains, the effect on the host and function in the human intestines remains largely unknown $(25,26)$. In light of our results, these topics represent further questions that should be explored.

Finally, in the present study, significantly lower levels of Bifidobacterium were not observed in the PH-G than in the Non-PH-G; in fact, they tended to be higher than in the non-PH-G ( $\mathrm{p}=0.063)$. In previous reports, it has been re- 
Table 4. A Comparison of the Gut Microbiota between the ALC and Non-ALC Groups in the PH-G (median \pm SD).

\begin{tabular}{lccc}
\hline & ALC & Non-ALC & p value \\
\hline Bifidobacterium & $13.71 \pm 2.593$ & $5.863 \pm 2.374$ & $0.042 *$ \\
Lactobacillales & $17.81 \pm 2.798$ & $9.634 \pm 3.517$ & 0.131 \\
Bacteroides & $38.50 \pm 3.257$ & $42.45 \pm 3.116$ & 0.391 \\
Prevotella & $1.909 \pm 1.909$ & $5.079 \pm 3.837$ & 0.572 \\
Clostridium cluster IV & $2.020 \pm 1.063$ & $3.938 \pm 1.029$ & 0.217 \\
Clostridium cluster XIVa & $7.561 \pm 2.848$ & $12.923 \pm 3.344$ & 0.283 \\
Clostridium cluster IX & $1.504 \pm 0.862$ & $2.186 \pm 1.283$ & 0.715 \\
Clostridium cluster XI & $0.149 \pm 0.099$ & $1.443 \pm 0.765$ & 0.259 \\
Clostridium cluster XVIII & $0.863 \pm 0.447$ & $1.102 \pm 0.523$ & 0.753 \\
others & $6.143 \pm 1.841$ & $7.714 \pm 1.913$ & 0.575 \\
\hline *statistically significant difference & & \\
SD: standard deviation, ALC: alcohol group, Non-ALC: non-alcohol group, PH- \\
G: portal hypertension group
\end{tabular}

ported that the Bifidobacterium genus reduces liver cirrhosis (27). However, it should be noted that in recent years, the levels of the Bifidobacterium genus have been reported to increase with both alcohol dependence and alcoholic liver cirrhosis (28-30). This is attributed to the fact that some species in the Bifidobacterium genus has an acetaldehyde cumulative effect (31). East Asian people have a genetic variation in alcohol metabolism involving frequently increased acetaldehyde levels after ethanol consumption, and they characteristically have a poor physiological reaction to alcohol (32). Bifidobacterium dentium is one species of Bifidobacterium found to be associated with liver cirrhosis; this species has been found to be able to produce large amounts of $\gamma$-aminobutyric acid, an important neurotransmitter (33). Based on these facts, it is also possible that there is compensatory feedback of the microbiota to changes in the metabolism of serotonin and other neural mediators in cases of alcohol dependence and alcoholic liver cirrhosis. The present study showed that $50 \%$ of the PH-G had alcoholic liver cirrhosis, and the levels of Bifidobacterium were significantly higher in the ALC group than in the Non-ALC group. Therefore, it is possible that the gut microbiota in the ALC group reflected an increase in Bifidobacterium genus levels associated with alcohol metabolism. In the future, we should conduct futher analyses on the etiology of cirrhosis, not only based on an individual's alcohol consumption, but using a large number of samples.

As the present findings revealed a significant increase in Lactobacillales and an increasing tendency in the genus Bifidobacterium, it may be necessary to reexamine the application of probiotic formulations based on these two genera. These probiotic preparations have anti-inflammatory effects, are useful drugs in the human intestine for protective action against pathogens, and are commonly used in the treatment of various diseases, including alcoholic liver disease $(34,35)$. However, given that this study showed an increase in Lactobacillales and Bifidobacterium in the gut microbiota of the PH-G, further supplementation of these mi- croorganisms may be harmful. Therefore, great care must be taken regarding the administration of probiotic formulations to these types of patients.

The present study showed no significant difference between the levels of Clostridium cluster XI and Clostridium cluster XIVa, but bile acids are known to be related to intestinal bacteria. Yoshimoto et al. found that serum levels of deoxycholic acid (DCA) and the composition of Clostridium cluster XI and XIVa that convert primary bile acids to DCA were increased in obese mice. DCA is said to produce reactive oxygen species that cause DNA damage and senescence-associated secretory phenotype (SASP) related to hepatocarcinogenesis (36). In cases of $\mathrm{PH}$, it has been reported that bile acids in the peripheral blood are increased due to leakage of superior mesenteric vein (SMV)-derived blood to the periphery due to an increase in portal venous shunt (37). It is necessary to pay close attention to the relationship between bile acids and intestinal bacteria going forward.

Several limitations associated with the present study warrant mention. First, this was a single-center study, which may limit the generalizability of the results. Second, the sample size was small, and this was a cross-sectional study. Third, we did not analyze the infection rate of Helicobacter pylori, which is known to affect the gut microbiota. Finally, PH was related to liver cirrhosis in all patients; thus, it was difficult to clearly distinguish $\mathrm{PH}$ and liver cirrhosis.

In conclusion, this exploratory study of the characteristics of the gut microbiota in $\mathrm{PH}$ patients with esophago-gastric varices due to liver cirrhosis showed an increase in Streptococcus and a decrease in short-chain fatty acids, such as butyric acid and propionic acid. Furthermore, the genus Bifidobacterium, which is said to be reduced by liver cirrhosis, did not show decreased levels. In the future, we will attempt to develop probiotic treatments following further analyses using methods that evaluate bacteria at the species level, such as the intestinal flora analysis method and next-generation sequencing. 
The authors state that they have no Conflict of Interest (COI).

\section{Acknowledgement}

We express our gratitude to Eri Yamauchi, Motoko Kawashima, Waki Nagashima, Yuki Nozaki, Atsuko Ishibashi, Chihiro Tanaka, Akiko Tanaka, and Tomoko Nagaura for their invaluable support.

\section{References}

1. Bellot P, Francés R, Such J. Pathological bacterial translocation in cirrhosis: pathophysiology, diagnosis and clinical implications. Liver Int 33: 31-39, 2013.

2. Guarner C, Soriano G. Bacterial translocation and its consequences in patients with cirrhosis. Eur J Gastroenterol Hepatol 17: 27-31, 2005.

3. Giannelli V, Di Gregorio V, Iebba V, et al. Microbiota and the gutliver axis: bacterial translocation, inflammation and infection in cirrhosis. World J Gastroenterol 20: 16795-16810, 2014.

4. Roderburg C, Luedde T. The role of the gut microbiome in the development and progression of liver cirrhosis and hepatocellular carcinoma. Gut Microbes 5: 441-445, 2014.

5. Liu WT, Marsh TL, Cheng H, Forney LJ. Characterization of microbial diversity by determining terminal restriction fragment length polymorphisms of genes encoding 16S rRNA. Appl Environ Microbiol 63: 4516-4522, 1997.

6. Wiest R, Lawson M, Geuking M. Pathological bacterial translocation in liver cirrhosis. J Hepatol 60: 197-209, 2014.

7. Teltschik Z, Wiest R, Beisner J, et al. Intestinal bacterial translocation in rats with cirrhosis is related to compromised Paneth cell antimicrobial host defense. Hepatology 55: 1154-1163, 2012.

8. Moghadamrad S, McCoy KD, Geuking MB, et al. Attenuated portal hypertension in germ-free mice: function of bacterial flora on the development of mesenteric lymphatic and blood vessels. Hepatology 61: 1685-1695, 2015.

9. Qin N, Yang F, Li A, et al. Alterations of the human gut microbiome in liver cirrhosis. Nature 513: 59-64, 2014.

10. Lu Y, Chen J, Zheng J, et al. Mucosal adherent bacterial dysbiosis in patients with colorectal adenomas. Sci Rep 6: 26337, 2016.

11. Nagashima K, Hisada T, Sato M, Mochizuki J. Application of new primer-enzyme combinations to terminal restriction fragment length polymorphism profiling of bacterial populations in human feces. Appl Environ Microbiol 69: 1251-1262, 2003.

12. Chavez-Tapia NC, Tellez-Avila FI, Garcia-Leiva J, Valdovinos MA. Use and overuse of proton pump inhibitors in cirrhotic patients. Med Sci Monit 14: CR468-CR472, 2008.

13. Bajaj JS, Riggio O, Allampati S, et al. Cognitive dysfunction is associated with poor socioeconomic status in patients with cirrhosis: an international multicenter study. Clin Gastroenterol Hepatol 11: 1511-1516, 2013.

14. Kang SH, Yim HJ, Kim SY, et al. Proton pump inhibitor therapy is associated with reduction of early bleeding risk after prophylactic endoscopic variceal band ligation: a retrospective cohort study. Medicine (Baltimore) 95: e2903, 2016.

15. Fasullo M, Rau P, Liu DQ, et al. Proton pump inhibitors increase the severity of hepatic encephalopathy in cirrhotic patients. World J Hepatol 11: 522-530, 2019.

16. Nardelli S, Gioia S, Ridola L, Farcomeni A, Merli M, Riggio O. Proton pump inhibitors are associated with minimal and overt hepatic encephalopathy and increased mortality in patients with cirrhosis. Hepatology 70: 640-649, 2019.

17. Janka T, Tornai T, Borbély B, et al. Deleterious effect of proton pump inhibitors on the disease course of cirrhosis. Eur J Gastroenterol Hepatol 32: 257-264, 2020.

18. De Roza MA, Kai L, Kam JW, et al. Proton pump inhibitor use increases mortality and hepatic decompensation in liver cirrhosis.
World J Gastroenterol 25: 4933-4944, 2019.

19. Okura Y, Namisaki T, Sato S, et al. Proton pump inhibitor therapy does not increase serum endotoxin activity in patients with cirrhosis. Hepatol Res 49: 232-238, 2019.

20. Yamamoto $\mathrm{K}$, Ishigami $\mathrm{M}$, Honda $\mathrm{T}$, et al. Influence of proton pump inhibitors on microbiota in chronic liver disease patients. Hepatol Int 13: 234-244, 2019.

21. Tang R, Wei Y, Li Y, et al. Gut microbial profile is altered in primary biliary cholangitis and partially restored after UDCA therapy. Gut 67: 534-541, 2018.

22. Furusawa Y, Obata Y, Fukuda S, et al. Commensal microbederived butyrate induces the differentiation of colonic regulatory $\mathrm{T}$ cells. Nature 504: 446-450, 2013.

23. Boursier J, Mueller O, Barret M, et al. The severity of nonalcoholic fatty liver disease is associated with gut dysbiosis and shift in the metabolic function of the gut microbiota. Hepatology 63: 764-775, 2016.

24. Atarashi $K$, Tanoue $T$, Shima $T$, et al. Induction of colonic regulatory $\mathrm{T}$ cells by indigenous Clostridium species. Science 331: $337-$ 341, 2011.

25. Ohue-Kitano R, Taira S, Watanabe $K$, et al. 3-(4-hydroxy-3methoxyphenyl) propionic acid produced from 4-hydroxy-3methoxycinnamic acid by gut microbiota improves host metabolic condition in diet-induced obese mice. Nutrients 11: E1036, 2019.

26. García-Sánchez JE, García-Sánchez E, Garcia-Moro M. The clinical microbiologist before the taxonomic changes in the genus Clostridium. Rev Esp Quimioter 29: 239-243, 2016.

27. Chen Y, Yang F, Lu H, et al. Characterization of fecal microbial communities in patients with liver cirrhosis. Hepatology 54: 562572, 2011.

28. Peng Y, Shi H, Qi X, et al. The ADH1B Arg47His polymorphism in East Asian populations and expansion of rice domestication in history. BMC Evol Biol 10: 15, 2010.

29. Wang W, Chen L, Zhou R, et al. Increased proportions of Bifidobacterium and the Lactobacillus group and loss of butyrateproducing bacteria in inflammatory bowel disease. J Clin Microbiol 52: 398-406, 2014.

30. Taur Y, Xavier JB, Lipuma L, et al. Intestinal domination and the risk of bacteremia in patients undergoing allogeneic hematopoietic stem cell transplantation. Clin Infect Dis 55: 905-914, 2012.

31. Wahlström A, Sayin SI, Marschall H-U, Bäckhed F. Intestinal crosstalk between bile acids and microbiota and its impact on host metabolism. Cell Metab 24: 41-50, 2016.

32. Leon DA, Saburova L, Tomkins S, et al. Hazardous alcohol drinking and premature mortality in Russia: a population based casecontrol study. Lancet 369: 2001-2009, 2007.

33. Masip L, Veeravalli K, Georgiou G. The many faces of glutathione in bacteria. Antioxid Redox Signal 8: 753-762, 2006.

34. Lovinger DM. Serotonin's role in alcohol's effects on the brain. Alcohol Health Res World 21: 114-120, 1997.

35. Pereg D, Kotliroff A, Gadoth N, Hadary R, Lishner M, Kitay-Cohen Y. Probiotics for patients with compensated liver cirrhosis: a double-blind placebo-controlled study. Nutrition 27: 177181, 2011.

36. Yoshimoto S, Loo TM, Atarashi K, et al. Obesity-induced gut microbial metabolite promotes liver cancer through senescence secretome. Nature 499: 97-101, 2013.

37. Hayashi H, Beppu T, Okabe H, et al. Combined measurements of serum bile acid level and splenic volume may be useful to noninvasively assess portal venous pressure. J Gastroenterol 47: 13361341, 2012.

The Internal Medicine is an Open Access journal distributed under the Creative Commons Attribution-NonCommercial-NoDerivatives 4.0 International License. To view the details of this license, please visit (https://creativecommons.org/licenses/ by-nc-nd/4.0/).

(C) 2020 The Japanese Society of Internal Medicine Intern Med 59: 2089-2094, 2020 